\title{
Balanced Approach to Neonates and Young Infants with Fever
}

\author{
Gihad Alsaeed $^{1,}$, Ibrahim Alsaeed $^{2}$, Mohamed Alsaeed ${ }^{3}$ \\ ${ }^{1}$ Department of Pediatrics, Altakhassussi Hospital, Riyadh, Saudi Arabia \\ ${ }^{2}$ Faculty of Medicine, University of Milan, Milan, Italy \\ ${ }^{3}$ Faculty of Medicine, University of Pavia, Pavia, Italy
}

\section{Email address:}

gehadalsaeed@yahoo.com (G. Alsaeed), ibrahimalsaeed955@gmail.com (I. Alsaeed),mohamed.alsaeed@universitadipavia.it (M. Alsaeed) ${ }^{*}$ Corresponding author

\section{To cite this article:}

Gihad Alsaeed, Ibrahim Alsaeed, Mohamed Alsaeed. Balanced Approach to Neonates and Young Infants with Fever. American Journal of Pediatrics. Vol. 5, No. 4, 2019, pp. 254-259. doi: 10.11648/j.ajp.20190504.25

Received: October 16, 2019; Accepted: November 7, 2019; Published: November 14, 2019

\begin{abstract}
Fever in early infancy may indicate the presence of an invasive bacterial infection [IBI]. Viral infection is the most common cause of fever in young infants, with Rhinovirus being the most common. Rectal temperature $38^{\circ} \mathrm{C}$ should be considered as fever. Bacterial infection may be the cause of fever in this age group even in the presence of a clear viral etiology. Evaluation and treatment of febrile infants during the first 3 months of life requires a balanced and cautious approach. After the introduction of vaccines in early infancy, there have been considerable changes in the bacterial pathogens and consecutive changes in the evaluation and empiric treatment of febrile young infants. While full septic screen seems necessary in the evaluation of many febrile young infants, partial septic screen without Lumbar puncture could be better in selected cases. It is the responsibility of the pediatrician to recognize the risk of invasive bacterial infection and to avoid unnecessary investigations at the same time. In this study, we suggest a simple approach that avoids the weak points of the available approach plans. Using clinical examples, we try to simplify this practical challenge.
\end{abstract}

Keywords: Febrile Infant, Invasive Bacterial Infection, Risk Factors, Full Septic Screen, Empiric Antibiotics

\section{Introduction}

\subsection{Definition of Fever}

A rectal temperature $(\mathrm{RT})$ of $38^{\circ} \mathrm{C}(100.4 \mathrm{~F})$ or greater is generally regarded as fever. Rectal temperature measurement using a digital electronic thermometer is the best method. Axillary, and infrared temporal or tympanic temperature measurements are less reliable in infants. Similarly, Oral pacifier temperature has low sensitivity for detection of fever $[1,2]$. However, a temperature of $38^{\circ} \mathrm{C}$ or greater by any measurement method should be considered as fever and confirmed by rectal temperature in the ER [3]. Rectal temperature measurement is contraindicated in immunodeficiency, when there is risk of bleeding, neutropenia, and in the case of necrotizing enterocolitis [4]. Axillary temperature can be used when rectal temperature is contraindicated or not possible. Because of the variability from device to device and by site of measurement, it is not recommended to alter the reported temperature by adding 0.5 $-1^{\circ} \mathrm{C}$ to estimate the rectal temperature [4].

\subsection{The Febrile Infant}

A Young infant should be considered febrile if he has a temperature of $38^{\circ} \mathrm{C}$ or more taken by a medical staff, or a temperature of $38^{\circ} \mathrm{C}$ or more taken by parents rectally (RT). A Temperature of $38^{\circ} \mathrm{C}$ taken at home by parents using other methods needs attendance to the ER for Rectal Temperature measurement. If rectal temperature (RT) is less than $38^{\circ} \mathrm{C}$, the history and physical examination are negative, and the baby did not take paracetamol at home, he should be considered afebrile and followed for 24 hours. If the infant was given paracetamol, looks ill, refuses feeding or there is a risk perinatal factor of infection, he should be considered febrile even if the rectal temperature in the ER is normal. 


\subsection{Causes of Fever in Young Infants}

$58 \%$ of febrile infants have viral infections and $15 \%$ have bacterial infections. Neonates less than 28 days of age with a viral infection remain at high risk for concomitant invasive bacterial illness $[5,6]$. Bundling should not be considered a cause of high RT $38^{\circ} \mathrm{C}$ when room temperature is less than 35 and humidity less than $75 \%$ [7]. Infants 29 to 90 days of age have a lower risk of invasive bacterial illnesses but remain at significant risk of urinary tract infections [8]. The incidence of invasive bacterial infection in febrile infants during the first month of life is $9-19 \%$ and decreases to $7-$ $11 \%$ during the second and third months $[1,2]$. Examples of Invasive bacterial infections (IBIs) are: bacteremia, sepsis, meningitis, pneumonia, gastroenteritis, pustulosis, cellulitis, septic arthritis, urinary tract infection (UTI) [6]. UTIs account for most bacterial infections in infants during the first 3 months of life [6].

\section{Febrile Infant Younger than 3 Months Approach (Figure 1)}

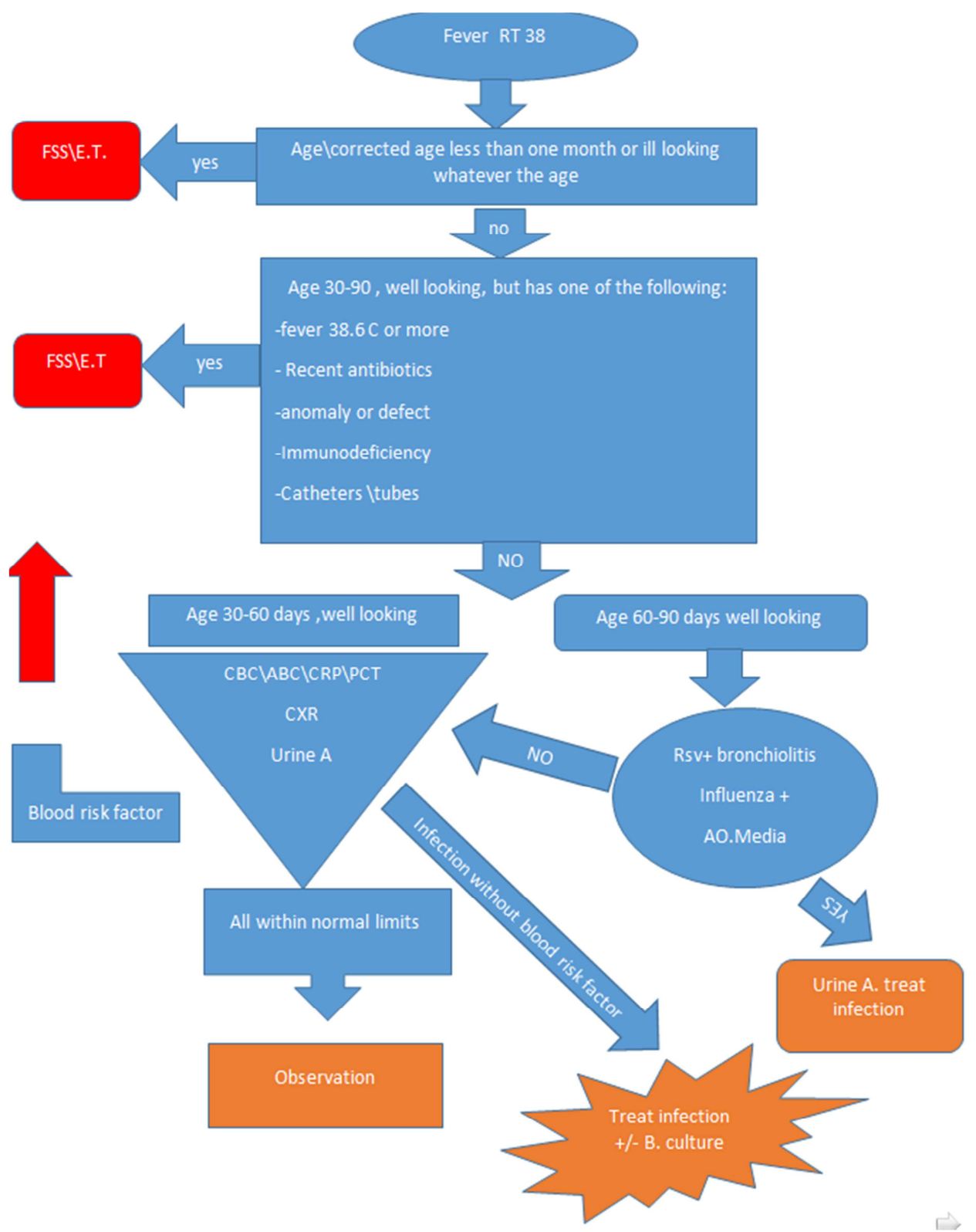

Figure 1. Febrile infant younger than 3 months Approach algorithm.

It is not easy to find a balance between a cost effective, noninvasive investigation policy and the need to recognize all cases of invasive bacterial infections in young infants. Considerable modifications were introduced on febrile young infant investigation and management plan after the wide introduction of Haemophilus influenza type B, and pneumococcal vaccines. A reasonable individual approach for each febrile young infant is the rule. However, the following common principles are recommended by most researchers: 
I -All febrile neonates younger than one month need direct admission for full septic screen and empiric treatment [9].

II-All febrile premature infants whose corrected age [(weeks - (40-gestational age) $]$ is less than one month need direct admission for full septic screen and treatment [10].

III-All ill appearing febrile young infants need direct admission for full septic screen and treatment. 10\% of ill appearing young infants (during the first 3 months) have an IBI [11].

IV- Well appearing febrile infants (30-90) days old:

It is the most common clinical scenario that needs Careful individual assessment for the presence of clinical or laboratory (IBI) indicators to put a suitable management plan [1].

Clinical indicators of IBI in febrile infant $[12,13,5]$ :

1. Age less than 28 days corrected age.

2. A Rectal temperature of $38.6^{\circ} \mathrm{C}$ or more.

3. Ill appearance. (Increased work of breathing, mottled skin, weak cry, irritability, lethargy, poor feeding, poor response, or limp).

4. Prematurity.

5. Not given the first immunizations at age 2 months.

6. Antibiotic treatment during the last 7 days.

7. Maternal infection risk, like fever or early membrane rupture or positive high vaginal swab.

8. Any possible indicator of herpes simplex infection (skin, eye, mouth vesicles, seizures, focal neurological findings, maternal herpes lesions).

9. Chromosomal defect, congenital anomaly.

10. tubes, or catheters in situ or used frequently.

Laboratory indicators of [IBI]:

1- WBC: 15000 or less than 5000 .

2- ABC (absolute band count) of 1500 or more.

3- CRP value more than $20 \mathrm{mg} / \mathrm{L}$.

4- PCT value more than $0.5 \mathrm{ng} / \mathrm{Ml}$.

5- A Chest $\mathrm{X}$ ray suggesting pneumonia.

6- Urine analysis (WBC:10 or more in field, nitrite positive, or positive gram stain).

\section{Suggested Approach for Well Appearing Febrile Infant 29-90 Days Old}

I- clinical assessment for the presence of the following risk factors:

1. RT $38.6^{\circ} \mathrm{C}$

2. Recent use of antibiotics

3. Immunodeficiency, catheters, chromosomal or congenital anomaly.

4. Indicators of herpes simplex [HSV] infection.

II- laboratory assessment for the presence of the following risk factors:

1. WBC, $\mathrm{ABC}$.

2. CRP, PCT

3. Urine analysis

4. Chest $x$ ray.
According to this primary evaluation, a febrile infant will be one of the following:

1. Well appearing febrile infant who has clear focus of infection (pneumonia, osteomyelitis, urinary tract infection, umbilicitis, cellulitis,.. etc) and has one or more clinical risk factor or blood risk factor( $\mathrm{WBC}$, ABC, CRP, PCT). This infant needs second step full septic screen including CSF and empiric treatment as an inpatient pending cultures [2].

2. Well appearing febrile infant who has clear focus of infection (UTI, pneumonia, skin infection, otitis media...... etc.) or viral infection like (bronchiolitis, influenza...etc.), but has no clinical risk factors and no blood test risk factor. This infant needs no further study and can be treated according to his infection as inpatient or outpatient $[11,2,14]$.

3. Well appearing febrile infant without clear focus of infection but has one or more clinical or laboratory risk factors. This infant needs full septic screen and empiric treatment pending cultures [2].

4. Well appearing febrile infant without clear focus of infection and without any risk factor. This infant needs observation and follow up after 24hours without antibiotics [15].

\section{Clinical Examples}

1. 50-day old baby boy has fever $38.2^{\circ} \mathrm{C}$ taken by mother using temporal thermometer. He looks well and has no clinical risk factors of infection. Primary evaluation disclosed: right upper lobe pneumonia. WBC 4000, crp $30 \mathrm{mg} / \mathrm{L}$.

What is the next step?

This baby needs full septic screen and empiric treatment for 2 days then adjust the dose and spectrum according to cultures. Pneumonia confirmed by CXR in the presence of abnormal blood tests in a well appearing infant 29-90 days is an important indication for CSF study (5\% of them have meningitis [3].

2. 42-day old baby girl previously well but today has a fever RT $38.5^{\circ} \mathrm{C}$, has no clinical risk factors, no clear focus of infection by physical examination. in her primary evaluation:

WBC: 14000, ABC: 1600, CRP:15mg/L. PCT:0.25, CXR: clear, urine test: WBC: 12 , nitrite + .

What is the next step?

This infant is well appearing, no clinical or blood risk factors. She has possible urinary infection [UTI]. Plan: urine culture using catheter or suprapubic, then discharge home on oral amoxicillin.

If urine analysis indicate infection (WBC:10 or more in field, nitrite positive or, positive urine gram stain) without any risk factor, it is indicated to ask for urine culture and start oral antibiotics [15]. A UTI in a well appearing infant 30-90 days of age who is febrile but the rectal temperature is less than $38.6^{\circ} \mathrm{C}$ and all the blood tests are normal and the chest $\mathrm{x}$ ray is normal, can be treated as 
outpatient and it is better to give the first dose of antibiotics parenterally [16].

3. 66-day old baby boy, previously well, has no clinical risk factors, his mother recorded $38.3^{\circ} \mathrm{C}$ temperature by tympanic measurement. In ER his RT: $38^{\circ} \mathrm{C}$, looks well, no clear focus of infection.

What is the next step?

This baby needs only urine test and observation. If the infant is older than 60 days, well looking and has no clear focus of infection and no clinical risk factor and his rectal temperature is less than $38.6^{\circ} \mathrm{C}$, it is recommended to take urine analysis and culture only [15].

4. 60-day old baby girl has fever $38.2^{\circ} \mathrm{C}$ and cough, no perinatal risk factors, her brother has flu like symptoms. She looks well with good oral intake, good hydration and stable in room air. RSV swab+.

What is the next step?

This baby needs urine culture, supportive treatment and observation. If the infant has documented viral infection like (RSV, influenza, rhino) and his rectal temperature is less than $38.6^{\circ} \mathrm{C}$ and looks well, only urine culture by catheter or suprapubic aspiration without antibiotics is recommended [14].

5. 46 day old baby boy, 34 week preterm, SVD, has fever $38.2^{\circ} \mathrm{C}$ and cough, his brother has flu like symptoms, he looks well with good oral intake, good hydration and stable in room air. RSV swab+.

What is the next step?

This baby needs full septic screen. (his corrected age: is 4 days). If the infant is ill appearing or less than 30 days of corrected age, full septic screen and antibiotics should be started even if a viral infection is well-documented [1].

6. The previous baby was admitted, full septic screen performed and empiric cefotaxime, ampicillin were started. No laboratory risk factors, and the CSF is clear. After 48 hours cultures are negative but the baby is still febrile.

What is the next step?

Discontinue antibiotics and follow up. If cultures are negative but the infant is still febrile, antibiotics should be discontinued and the infant followed as inpatient in the hospital till the fever has been resolved or diagnosed and treated properly [16].

7. 60-day old baby girl has fever [RT] $38.5^{\circ} \mathrm{C}$. After her vaccine visit 5 days ago she had runny nose and mild cough without fever for 4 days, then fever started with excessive crying, no risk factors, $\mathrm{P} / \mathrm{E}$ : red bulging tympani in the right ear.

What is the next step?

This baby needs urine analysis and culture, and start oral amoxicillin. If the baby 30-90 days old, has focal infection like otitis media or skin infection or UTI, looks well and no fever more than $38.6^{\circ} \mathrm{C}$, and blood tests are normal, it is recommended to collect urine for culture then treat the infection with antibiotics [1]. The risk of IBI in infants younger than 90 days of age is not increased by the presence of acute otitis media [AOM]. Evaluation and treatment should be based on other clinical features. Obtain urinalysis and urine culture alone or with a blood culture prior to administration of oral antibiotics (eg, amoxicillin). This approach may be most appropriate for infants closer to 60 days of age with rectal temperature $\geq 38.6^{\circ} \mathrm{C}\left(101.5^{\circ} \mathrm{F}[17\right.$, $18]$.

All focal infections without risk factors in well appearing infants older than one month can be treated without full septic screen [16].

8. 63-day old baby girl has fever $38.2^{\circ} \mathrm{C}$ today after her first vaccine yesterday, previously well, no clear focus of infection, looks well. This baby needs urine test and observation for 48hours.

Febrile infant who received his first vaccine within the last 48 hours, well looking, temperature less than $38.6^{\circ} \mathrm{C}$, and no clear focus of infection is recommended to order urine analysis and culture and follow up after $24 \mathrm{hrs}$ or at any time if he becomes ill appearing or have high temperature $38.6^{\circ} \mathrm{C}$ or more.

Febrile infant after his first vaccine who has high fever or ill appearance needs admission and full septic screen and empiric treatment $[2,16]$

\section{Management of Ill Appearing Febrile Young Infants and Neonates Younger Than One Month [16]}

i. Stabilize and manage septic shock if present.

ii. Full septic screen including cultures and LP.

iii. Empiric antimicrobials.

iv. Admission in the hospital.

$\mathrm{v}$. If any clinical indicator of HSV is present acyclovir should be started and investigations for HSV including viral cultures and PCR from lesion swab, blood and CSF is mandatory.

\section{Full Septic Screen}

Full septic screen should include all of the following:

1. Blood tests: $\mathrm{CBC}+\mathrm{CRP}+\mathrm{PCT}+\mathrm{B}$. culture + B. Sugar + B. calcium + Lactate + PT.PTTK + Fibrinogen + ALT + Bilirubin.

2. Urine analysis and culture (collected by catheter or suprapubic aspiration).

3. Stool culture (if WBC in diarrhea is more than 5 per field by microscopic examination)

4. CSF study: cytology, protein, sugar, gram stain, PCR for herpes simplex, culture.

5. Surface Swab culture from: conjunctiva, mouth, nasopharynx, and rectum (in one swab if possible).

6. Skin lesion swab cultures.

7. Chest $x$ ray

8. Abdominal ultrasound if there is UTI or chromosomal defect or anomaly. 


\section{Empiric Antimicrobials for Ill Appearing Febrile Infants According to Age}

1. 0-28 days: ampicillin+ cefotaxime +acyclovir. add vancomycin if there is septic shock or skin infection.

2. 29- 60 days: ceftriaxone or cefotaxime+ ampicillin + vancomycin. add acyclovir if needed.

3. 61-90 days: ceftriaxone + vancomycin.

\section{Empiric Antimicrobials for Well Appearing Febrile Infants According to Age [16]}

1. 0-28 days: ampicillin + cefotaxime

2. 29-90 days: ceftriaxone or cefotaxime.

After 48 hours empiric antimicrobials can be narrowed or discontinued according to culture results.

If the febrile infant (older than 30 days) had received antibiotics at home, full septic screen including LP and cultures is recommended. If he is well looking without any clinical or laboratory risk factors and normal chest $\mathrm{x}$ ray and urine test, he can be observed in the hospital without treatment. If any risk factor is present, the infant should be admitted and treated even if he looks well till the cultures are negative after 48 hours. An alternative approach for patients with a single risk factor of a temperature $\geq 38.6^{\circ} \mathrm{C}\left(101.5^{\circ} \mathrm{F}\right)$, antibiotic therapy in the past seven days, or one of the maternal risk factors is to give a single dose of intramuscular (IM) ceftriaxone (50 $\mathrm{mg} / \mathrm{kg}$ ) and discharge the patient to home with assured follow-up within 24 hours for a second IM dose of ceftriaxone, pending culture results [19].

\section{Conclusion}

Rectal temperature $38^{\circ} \mathrm{C}$ should considered as fever. Axillary temperature measurement can be accepted when rectal temperature is contraindicated. tympanic, temporal or pacifier temperature measurements are much less reliable in young infants. After the introduction of vaccines in early infancy, there have been considerable changes in the bacterial pathogens and consecutive changes in the evaluation and empiric treatment of febrile young infants. Urinary tract infection is the most common bacterial infection and the main source of IBI in this age group [12]. E.coli and group B streptococcus pyogenes (GBS) are the most common bacteria that may cause infection in young infants, followed by Listeria, and Staph Aureus [1, 12]. Febrile neonates younger than 28 days of age with clear viral infection (influenza, bronchiolitis, and bronchitis) remain at a substantial risk of IBIs and need a full septic screen [17-18]. Individual evaluation of each case to recognize the possible risk factors of invasive bacterial infection should be the first step in a successful management plan. To do a partial or complete septic screening, decision should be taken considering the infant's age, appearance, and risk factors. The suggested algorithm approach has been put to avoid the weak points of the available ones. However, further studies to explore its accuracy are needed.

\section{References}

[1] Greenhow TL, Hung YY, Pantell RH. Management and outcomes of previously healthy, full-term, febrile infants ages 7 to 90 days. Pediatrics. 2016.

[2] hannah F smitherman CGM. No Title. febrile infant younger than 90 days outpatient evaluation. Uptodate literature review : June 18th, 2019.

[3] Hui C, Neto G, Tsertsvadze A, Yazdi F, Tricco AC, Tsouros S, et al. Diagnosis and management of febrile infants $(0-3$ months). Evidence report/technology assessment. 2012.

[4] Callanan D. Detecting fever in young infants: Reliability of perceived, pacifier, and temporal artery temperatures in infants younger than 3 months of age. Pediatr Emerg Care. 2003.

[5] Mahajan P, Browne LR, Levine DA, Cohen DM, Gattu R, Linakis JG, et al. Risk of Bacterial Coinfections in Febrile Infants 60 Days Old and Younger with Documented Viral Infections. J Pediatr. 2018.

[6] Yarden-Bilavsky H, Ashkenazi-Hoffnung L, Livni G, Amir J, Bilavsky E. Month-by-month age analysis of the risk for serious bacterial infections in febrile infants with bronchiolitis. Clin Pediatr (Phila). 2011.

[7] Grover G, Berkowitz CD, Lewis RJ, Thompson M, Berry L, Seidel J. The effects of bundling on infant temperature. Pediatrics. 1994.

[8] Grohskopf L, Uyeki T, Bresee J, Cox N, Bridges C. Prevention and control of influenza with vaccines: Recommendations of the Advisory Committee on Immunization Practices (ACIP), 2011. Am J Transplant. 2011.

[9] Baker MD, Avner JR, Bell LM. Failure of infant observation scales in detecting serious illness in febrile, 4- to 8-week-old infants. Pediatrics. 1990.

[10] Gómez B, Mintegi S, Benito J, Egireun A, Garcia D, Astobiza E. Blood culture and bacteremia predictors in infants less than three months of age with fever without source. Pediatr Infect Dis J. 2010.

[11] Gomez B, Mintegi S, Bressan S, Da Dalt L, Gervaix A, Lacroix L. Validation of the "step-by-step" approach in the management of young febrile infants. Pediatrics. 2016.

[12] Fortunov RM, Hulten KG, Hammerman WA, Mason EO, Kaplan SL. Evaluation and treatment of community-acquired Staphylococcus aureus infections in term and late-preterm previously healthy neonates. Pediatrics. 2007.

[13] Maniaci V, Dauber A, Weiss S, Nylen E, Becker KL, Bachur R. Procalcitonin in young febrile infants for the detection of Serious bacterial infections. Pediatrics. 2008. 
[14] Kuppermann N, Dayan PS, Levine DA, Vitale M, Tzimenatos L, Tunik MG, et al. A Clinical Prediction Rule to Identify Febrile Infants 60 Days and Younger at Low Risk for Serious Bacterial Infections. In: JAMA Pediatrics. 2019.

[15] Velasco R, Benito H, Mozún R, Trujillo JE, Merino PA, Santiago, et al. Febrile young infants with altered urinalysis at low risk for invasive bacterial infection. A Spanish pediatric emergency research network's study. Pediatr Infect Dis J. 2015.

[16] hannah F smitherman CGM. febrile infant younger than 90 days managment. Uptodate literature review: May 23rd, 2019.
[17] Turner D, Leibovitz E, Aran A, Piglansky L, Raiz S, Leiberman A, et al. Acute otitis media in infants younger than two months of age: Microbiology, clinical presentation and therapeutic approach. Pediatr Infect Dis J. 2002.

[18] Nozicka CA, Hanly JG, Beste DJ, Conley SF, Hennes HM. Otitis media in infants aged 0-8 weeks: Frequency of associated serious bacterial disease. Pediatr Emerg Care. 1999.

[19] Schwartz S, Raveh D, Toker O, Segal G, Godovitch N, Schlesinger Y. A week-by-week analysis of the low-risk criteria for serious bacterial infection in febrile neonates. Arch Dis Child. 2009. 